\title{
Downregulated microRNAs in the colorectal cancer: diagnostic and therapeutic perspectives
}

\author{
Rosa Hernández ${ }^{1,2,3}$, Ester Sánchez-Jiménez ${ }^{4}$, Consolación Melguizo ${ }^{1,2,3}$, Jose Prados ${ }^{1,2,3, *}$ \& Ana Rosa Rama ${ }^{1,5}$ \\ ${ }^{1}$ Institute of Biopathology and Regenerative Medicine (IBIMER), Center of Biomedical Research (CIBM), University of Granada, \\ Granada 18100, ${ }^{2}$ Biosanitary Institute of Granada (ibs. GRANADA), SAS-Universidad de Granada, Granada 18100, ${ }^{3}$ Department of \\ Human Anatomy and Embryology, School of Medicine, University of Granada, Granada 18100, ${ }^{4}$ Proteomics Laboratory CSIC/UAB, \\ Institute of Biomedical Research, Barcelona 08036, ${ }^{5}$ Department of Health Science, University of Jaén, Jaén 23071, Spain
}

Colorectal cancer (CRC), the third most common cancer in the world, has no specific biomarkers that facilitate its diagnosis and subsequent treatment. The miRNAs, small single-stranded RNAs that repress the mRNA translation and trigger the mRNA degradation, show aberrant levels in the CRC, by which these molecules have been related with the initiation, progression, and drug-resistance of this cancer type. Numerous studies show the microRNAs influence the cellular mechanisms related to the cell cycle, differentiation, apoptosis, and migration of the cancer cells through the post-transcriptionally regulated gene expression. Specific patterns of the upregulated and down-regulated miRNA have been associated with the CRC diagnosis, prognosis, and therapeutic response. Concretely, the downregulated miRNAs represent attractive candidates, not only for the CRC diagnosis, but for the targeted therapies via the tumor-suppressing microRNA replacement. This review shows a general overview of the potential uses of the miRNAs in the CRC diagnosis, prognosis, and treatment with a special focus on the downregulated ones. [BMB Reports 2018; 51(11): 563-571]

\section{miRNAs: CHARACTERISTICS AND BIOGENESIS}

The micro-ribonucleic acids (RNAs), or miRNAs, are posttranscriptional regulatory elements consisting of 17-25 short nucleotides and single-strand and noncoding RNAs (1) that are highly conserved between the eukaryotic cells, animals, and plants related to numerous pathophysiological processes (2). The miRNAs are essential for biological processes such as the signal transduction, development, and cell growth and death

*Corresponding author. Tel: +34-958248819; Fax: +34-958246296; E-mail: jcprados@ugr.es

https://doi.org/10.5483/BMBRep.2018.51.11.116

Received 24 May 2018, Revised 12 June 2018, Accepted 10 July 2018

Keywords: Biomarkers, Colorectal cancer, Diagnosis, miRNA, Therapeutic target
(3). These processes are regulated by the binding of the miRNAs to the seed region of specific messenger RNAs (4) that represses their translation or degrades them. The seed region is located in the 3'-UTR extreme of the mRNAs, consisting of a sequence of 2-8 nucleotides repeated in different genes, thereby allowing the targeting of several ones by the miRNAs (2).

The miRNAs are generated from the primary transcript (pri-miRNA) in the nucleus, where the RNase III Drosha and the double-strand RNA binding protein, DGCR8 (microprocessor complex), cut its flanked simple strands (5). The result is the 65-nucleotides-hairpin, called the miRNA precursor (or the pre-miRNA), and it is moved to the cytoplasm using the exportin (6). The dicer cuts the pre-miRNA near the terminal bulge, generating a 22-bp mature miRNA duplex. The Argonaute 2 protein (AGO2) binds the pre-miRNA to form the miRNA-induced silencing complex, or the miRISC (7), that is able to select the leading strand that will be responsible for targeting the regulating mRNAs (Fig. 1).

\section{miRNAs IN THE COLORECTAL CANCER}

The miRNAs are usually deregulated in almost all the cancer types. Approximately $50 \%$ of the alterations are located in the cancer-associated genomic regions or fragile sites, leading the cells to act abnormally or aberrantly (8), thereby suggesting they play a vital role as the oncogenes or the tumor-suppressor genes. Lu et al. (9) show a bigger proportion of the downexpressed miRNA-217 in the tumor tissues than in the healthy tissues, and this can be used as the miRNA signature in the cancer diagnosis. In addition, they can be found in many different body fluids such as the bronchial lavage, seminal liquid, tears, breast milk, and amniotic cerebrospinal, pleural, or peritoneal fluids (10). The miRNAs resist the degradation (including in the lipid vesicles), interacting with the plasma protein, and traveling in the exosomes, microvesicles, or apoptotic bodies (11).

The colorectal cancer (CRC) is a heterogeneous disease with genetic and localization differences, which are highly related to the patient lifestyle and environment. The risk factors

ISSN: 1976-670X (electronic edition)

Copyright (c) 2018 by the The Korean Society for Biochemistry and Molecular Biology

(c) This is an open-access article distributed under the terms of the Creative Commons Attribution Non-Commercial License (http://creativecommons.org/licenses/by-nc/4.0) which permits unrestricted non-commercial use, distribution, and reproduction in any medium, provided the original work is properly cited. 


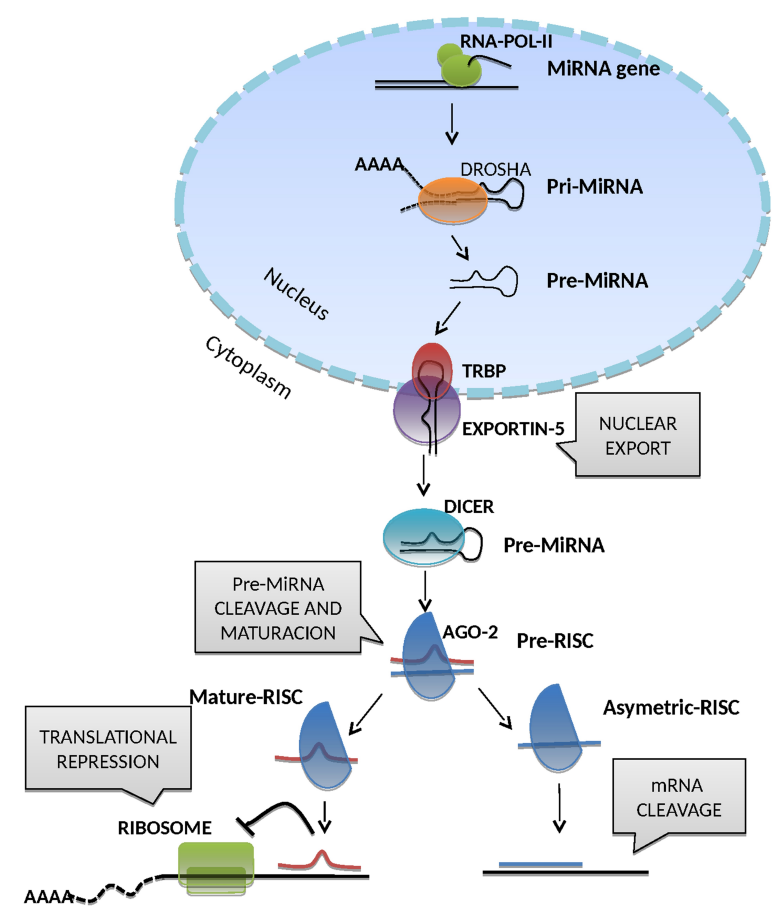

Fig. 1. miRNAs: characteristics and biogenesis. The miRNAs are transcribed by the polymerase II into the primary transcripts (pri-miRNAs) that are cleaved by the Drosha. This processing drives the formation of the hairpin precursor (pre-miRNAs). Exportin 5 transports the pre-miRNAs to the cytoplasm, where the Dicer processes them into the miRNA duplexes. One strand of the duplex (mature miRNA) is incorporated into the RNA-induced silencing complex (RISC), and it binds to the 3'-UTR of the target $\mathrm{mRNA}$, resulting in its degradation or translational repression.

include the hereditary mechanism, polyp formation, large-bowel inflammatory diseases, high-fat diet, physical inactivity, alcoholism, smoking, and obesity (12). Even though the CRC is one of the most common cancers worldwide (13), there are no valid biomarkers that facilitate the diagnosis and subsequent treatment.

The CRC is correlated to the inactivation of the tumorsuppressor genes, and the activation of the oncogenic signaling, amplifications, or mutations of the miRNA loci resulting mostly from the epigenetic alterations (4). The miRNAs may play a role in the physiological and pathological processes by influencing the cancer-stem-cell biology, angiogenesis, epithelial-mesenchymal and mesenchymal-epithelial transitions, or drug resistance (14). Furthermore, the colon-cancer stem cells have been identified as a contributor to the chemotherapy resistance; this is due, among other characteristics, to their plastic nature that lets them switch between the cancer nonstem cells and the cancer stem cells to avoid the chemotherapy effects (15).

The miRNA-sequencing studies regarding the CRC have determined specific expression profiles that could be related to the clinical-pathology and patient prognostics (16). Hamfjord et al. (8) identified 19 downregulated and 18 upregulated miRNAs in the CRC. Schee et al. (17) identified the five most expressed miRNAs (miRNA-10a-5p, -21-5p, -22-3p, -143-3p, and $-192-5 p$ ) inside a pool of 523 miRNAs from 88 CRC samples. The miRNA-143-3p and miRNA-192-5p are part of clusters related with the oncogenes, deoxynucleic acid (DNA)-repair genes, and genes from the WNT and MAPK signaling paths. Tokarz and Blasiak (4) found the miRNA-221 inhibits the angiogenesis activity through its binding to the c-Kit, Stat-5A, endothelial nitric oxide synthase, and ETS1 mRNAs; the miRNA-29a is associated with the cell -cycle arrest; the miRNA-21 leads to the tumor initiation, increasing the invasion and the metastasis; and the miRNA-26b is related to the cancer-cell growth.

\section{REMARKABLE miRNAs DOWNREGULATED IN THE COLORECTAL CANCER}

A remarkable number of miRNAs exhibit the differential expression in the CRC tissues with the downregulated miRNAs (Table 1), and this is especially relevant to the cell proliferation, apoptosis, and metastasis (Fig. 2). Some of these miRNAs has been associated with the CRC risk, patient survival, or treatment outcome.

The miRNA $16-1$ is frequently deleted or downregulated in many cancer-cell lines and various tumor tissues. It is usually transcribed with the miRNA 15a (miRNA-15a/16-1) and plays a role in the epithelial-mesenchymal transition, contributing to the metastasis capacity of the CRC cells (18). The p53 activates the miRNA-15a/16-1 that inhibits the expression of the AP4 (activating enhancer binding protein 4), a transcription factor that mediates the epithelial-mesenchymal transition; this leads to the metastasis repression in the lung, which is one of the most frequent produced by CRC (19). The tissue microarray of 90 patients with the CRC correlated with both the lower expressions of the miRNA15A and miRNA16-1 and a greater number of the IgA $+B$ cells, along with the shorter survival times of the patients. Liu et al. found the murine model of the primary CRC with the miRNA15A and miRNA16-1 deficiencies promoted the IgA-positive immunosuppressive B-cell (IgA+B) accumulation in the neoplastic tissues, and this was correlated with the more rapid tumor growth (20).

The ectopic expression of the miRNA-15a/16-1 raised the number of the G2/M phase cells in the CRC cell lines, reducing the colony formation and the tumor-induced mice. The CCNB1 (cyclin B1) protein levels were inversely correlated with the levels of the miRNA-15a/16-1, implying the CCNB1 is the target of these miRNAs (21). The CCNE1 (cyclin E1) comprises two miRNA-16-1 target sites, but a study wherein the small interfering RNA (siRNA) was observed against the CCNE1 shows the lower inhibition of the CCNE1 level compared to when the miRNA-16-1 level is increased, 
Table 1. Downregulated miRNAs in CRC Samples

\begin{tabular}{|c|c|c|c|}
\hline miRNA & Origin of sample & Biomarker & Reference \\
\hline miRNA-106a & Blood (plasma) & Prognosis and survival biomarker & (92) \\
\hline miRNA-126 & Blood (plasma) & $\begin{array}{l}\text { Decrease sensitivity to capecitabine and } \\
\text { oxaliplatin (XELOX) }\end{array}$ & (92) \\
\hline miRNA-137 & Cancer stem cells and normal colon stem cells & Capacity to suppress the tumorigenicity & (93) \\
\hline miRNA-143 & $\begin{array}{l}\text { CRC tissue, along with the corresponding normal mucosa } \\
\text { specimens }\end{array}$ & Increased response to 5 -fluoracil & (94) \\
\hline miRNA-16.1 & Human colon tumors and histologically normal tissue & Stage of CRC and tumorogenesis biomarker & (23) \\
\hline miRNA-29 & $\begin{array}{l}\text { Normal human colon epithelial cell lines and CRC cell } \\
\text { lines }\end{array}$ & Diagnostic biomarker & (95) \\
\hline miRNA-34 & $\begin{array}{l}\text { Formalin-fixed paraffin-embedded human CRC tissue and } \\
\text { normal colonic mucosa }\end{array}$ & $\begin{array}{l}\text { Prognosis biomarker Resistance to 5-fluorouracil } \\
\text { in treatment }\end{array}$ & (50) \\
\hline miRNA-365 & Human CRC tissue and non-neoplastic mucosa tissue & Progression and survival biomarker & $(40)$ \\
\hline miRNA-433-3p & Human CRC and normal human colon epithelial cells & CRC development biomarker & (96) \\
\hline miRNA-497-5p & CRC tissue relative to paired adjacent normal mucosa & Malignancy CRC biomarker & (97) \\
\hline miRNA-675 & Primary CRC tissue and paired adjacent non-tumor tissue & $\begin{array}{l}\text { Proliferation, invasion and migration related } \\
\text { biomarker }\end{array}$ & (98) \\
\hline miRNA-601 & Plasma & Diagnostic biomarker & (99) \\
\hline miRNA-760 & Plasma & Diagnostic biomarker & (99) \\
\hline
\end{tabular}

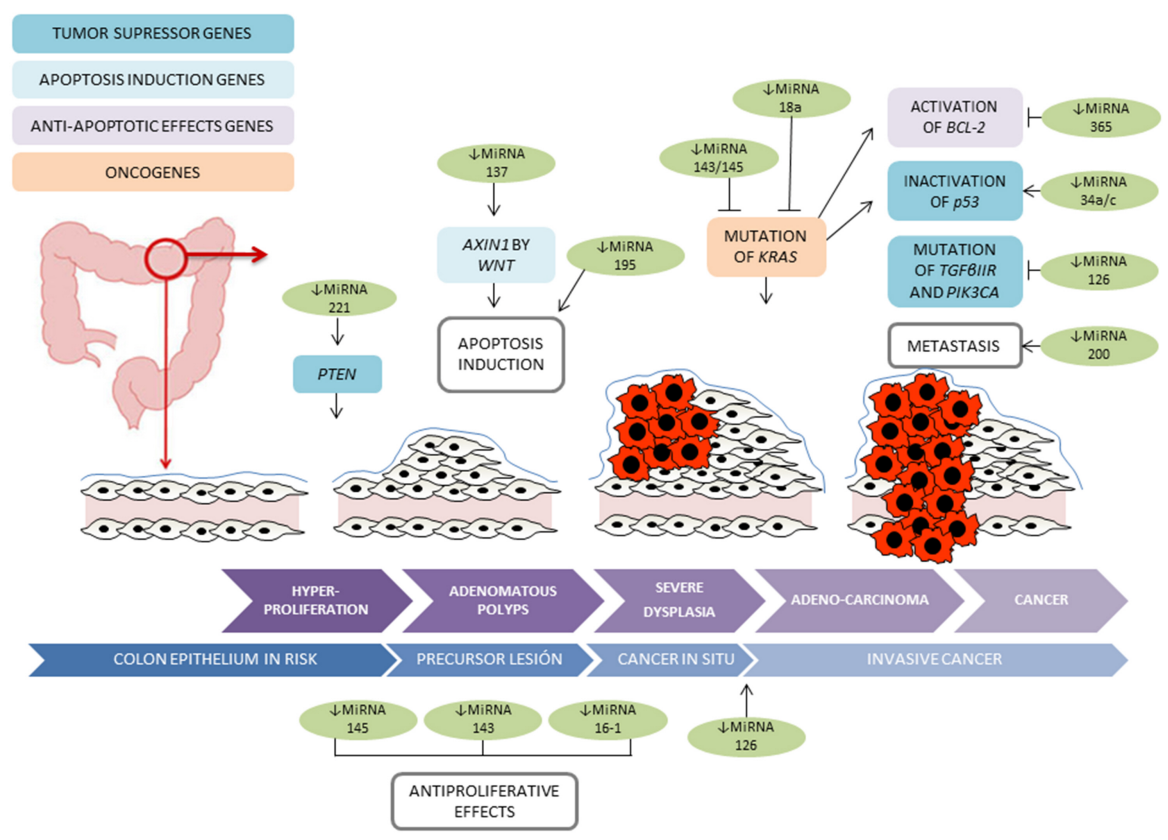

Fig. 2. Interactions of the miRNAs downregulated in the colorectal cancer with the intracellular signaling networks. The particular signaling pathways affected by the miRNAs are described in the review. MACC1, metastasis associated with the colon cancer-1; ErK5, extracellular-signalregulated kinase 5; TGF $\beta$ IIR, transforming growth factor- $\beta$ type-Il receptor; PIK3CA, phosphatidylinositol-4,5-bisphosphate 3-kinase catalytic subunit alpha; and PTEN, phosphatase and tensin homolog. suggesting other miRNA-16-1 targets are involved (1). The MiRNA-16-1 is inversely correlated with the cyclooxygenase-2 (COX-2), whose overexpression is a critical step of the CRC tumorigenesis (22). In the healthy cells, the miRNA-16-1 can bind to both the COX-2 target sequences and mediates its decay; however, in the tumor cells, this mechanism is inhibited by the HuR, which binds to the miRNA-16-1, allowing the COX-2 level to increase (23).

The cluster miRNA-143/miRNA-145 is a tumor suppressor that is usually downregulated in several tumors but is not expressed in the epithelial cells (24-27), while it is noticeably downregulated in the metastatic tumors of the CRC patients 
(28); its targets include the apoptosis inhibitor 5, K-RAS, ERK5, and insulin-receptor substrate 1. Drebber et al. (29) studied the methylation profile of the cluster miRNA-143/miRNA-145 before and after the chemoradiotherapy of 40 CRC patients, exhibiting a significant desregulation of these miRNAs in the post-therapeutic tissues. Li et al. (30) corroborated the full downregulation of the miRNA-145 expression in the CRC tissues, with it being 4-to-5-fold higher in the normal colonic tissues. Besides, the miRNA-145 overexpression decreased the migratory ability of the human CRC cells, indicating the miRNA-145 can suppress CRC cell migration and distant invasion.

Xu et al. (31) found a negative fold change in the CRC stages II, III, and IV with respect to the miRNA-14. A study of the miRNA-145 lipid-nanoparticles used in the mouse-xenograft tumors inhibited the tumor growth, and this is accompanied by a higher apoptosis and a lower proliferation (32). Ibrahim et al. (33) confirmed the miRNA-145 decreases the cellular growth and the tumor volume in mice; moreover, this corresponds with the miRNA downexpression in 145 of the targets, such as the ERK5 and the c-Myc. Tanaglu et al. (34) analyzed 16 miRNA-expression profiles of 40 patients with the recurrent and nonrecurrent CRC, finding the miRNA-145 expression was downregulated, suggesting its capacity as a sound biomarker for the early detection of this disease (35).

The miRNA-365 is often downregulated and involved in the regulation of the cell proliferation, differentiation, and apoptosis in numerous cancers cells, like those of the CRC (36-39). Nie et al. (40) demonstrated the correlation of the Cyclin D1 and Bcl-2 with the miRNA-365, as well as its downregulation to the prognosis in CRC patients. Zhou et al. (41) used microarrays to explore the possibly downregulated miRNA-mRNA pairs in The Cancer Genome Atlas and their regulatory roles in the CRC, corroborating that the miRNA-365-2 acts as the negative regulators of the $\mathrm{Bcl}-2$ protein. Another analysis of the expression array in the Caco- 2 cells displayed the suppression of the Mybl2 protein by the miRNA-365 (42).

The miRNA-34 a, b, and c are three similar members of the miRNA family with the same targets (43) and are regulated by the p53 and the DNA hypermethylation $(44,45)$. Their ectopic expression induces the senescence, apoptosis, and inhibition of the tumor-cell invasion (44), while the loss of the expression produces the resistance against the p53-induced apoptosis. The deregulated miRNA-34 family and the CpG methylation have been associated with the prognosis in the CRC and several other tumors (46). Gao et al. (47) found the downregulation of the miRNA-34a in the CRC tissues, while its expression is positively correlated with the disease-free survival; however, increased miRNA-34b/c is related to a poor prognosis (48).

The miRNA-34 ectopic expression inhibited the invasion, cell growth, and p53 activity in the HCT116 cells, but not in the knockout cells. The same results were obtained with the xenograft knockout, implying the expression is a prognostic marker for the CRC recurrence. A study of the nitric-oxide stress-induced cellular apoptosis, resulting from the p53dependent miRNA-34 overexpression, increased the resistance of the CRC cells to the apoptosis (49). Roy et al. (50) compared the miRNA-34 expression in formalin-fixed paraffinembedded human CRC tissues with those in the normal colonic-mucosa tissues; here, the miRNA-34 family is downregulated in both the cell cultures and the fixed tissues, which are involved in the growth and metastasis processes. Further, the CRC cells treated with the 5-aza-2'-deoxycytidine, a methyltransferase inhibitor, show a decrease of the miRNA-34 level related to the promoter hypermethylation, while the studies in vitro with the difluorinated curcumininduced re-expressed miRNA-34 show a consistency with the inhibition of the tumor-cell growth.

The miRNA-137 is embedded in the CpG island and is frequently silenced by the methylation in several tumors. The 5-azacytidine (5-AZA) was used in the CRC cell lines exhibiting a significant demethylation and inducing the miRNA-137 upregulation, suggesting its epigenetically silenced through the promoter methylation, which is not found or has very low levels in the healthy tissues. The restauration of the miRNA-137 levels reduced the cell proliferation, proposing a tumor-suppressor function. Svoboda et al. (51) found significant increases of the miRNA-137- and miRNA-125bexpression levels after the radiotherapy and the chemotherapy with the capecitabine, and this is associated with a worse chemotherapy response.

An inverse correlation between the miRNA-137 and the lysine (k)-specific demethylase 1A (LSD1), which participates in the maintenance of the global DNA methylation (52), has been found in the CRC cells (53). Also, the LSD1 overexpression has been documented with respect to several cancers (54). The downregulated miRNA-137 in the CRC cells causes the overexpression of the paxillin (PXN) gene, which encodes for a focal adhesion molecule (55), thereby involving a larger tumor size, an adverse differentiation status, a more extensive lymph-node invasion, a higher TNM stage, poor overall survival, a less favorable prognosis, a more proliferative ability, and a higher colony-formation capacity; similarly, this occurs with the Formin-like 2 (FMNL2), a target of the miRNA-137. Furthermore, the FMNL2-promoted proliferation, motility, and invasion of the CRC cell and metastasis in vivo was achieved by inducing the epithelialmesenchymal transition. The miRNA-137 ectopic expression inhibits the FMNL2 effects, allowing for the decreased proliferation and invasion by the CRC cells, and the metastasis to the liver and the intestine by the CRC xenografts (56).

Finally, the miRNA-143, an miRNA with the tumorsuppressor functions, is downregulated in several cancer types. The Ng et al. (57) study shows the ectopic miRNA-143 in the CRC cells reduced the DNA metyltransferases 3A (DNMT3A) expression and decreased the cell growth, malignant transformation phenotypes, and clone-formation efficiency. Similar 
findings were shown for the KRAS (58), a negative predictor for the EGFR-targeted therapies in the metastatic CRC, but not for the KRAS wild-type status. The miRNA-143 is an independent negative prognostic factor for the cancer-specific survival in the CRC KRAS wild-type patients (59), and its increased stable expression is associated with a low resistance (60). The miRNA-143 downregulates proteins such as the extracellular-regulate protein kinase 5 (ERK5), nuclear factor- $\mathrm{KB}(\mathrm{NF}-\mathrm{\kappa B})$, and $\mathrm{BCl}-2$, which are further reduced after the 5-fluorouracil (5-FU) exposition, causing a decreased viability and an increased cell death (60). Besides, the miRNA-143 targets the insulin-like growth factor 1 receptor (IGF-IR), inhibiting the oxaliplatin sensitivity (61).

\section{miRNAS AS BIOMARKERS IN THE COLORECTAL CANCER}

The CRC miRNAs are differentially expressed in diverse tissues and are potential biomarkers. Liu et al. (62) analyzed the miR-21 and the miR-92a concentrations in the CRC-patient serum, finding higher levels compared with that of the healthy subjects. Usually, high miRNA-21 levels are found in the plasma of the cancer patients (63). Low levels of the mir-150 can help distinguish between the patients with the advanced CRC and those with the adenomas (64). Recently, the miRNA-1290 has been proposed as a novel biomarker for the early detection of the CRC (65). Further, plasma panels can be used to quickly identify the polyps in a study of the miRNA concentrations (mir-532-3p, -331, -195, -17, -142-3p, -15b, -532 and -562 ) or the CRC stage IV (mir-431, -15b and -139-3p) (66). Even a chip has been designed for the analysis of the miR-9, -29b, -127-5p, -138, -143, -146a, -222, and -938, serving as a stool for the study of the progress of the disease using blood samples (67). Despite all these data, the following issues remain: A deficiency exists regarding the clinical information, a poor choice of the healthy controls, ethnicity is a potential confounding variable, and the small size of the experimentation groups do not allow for an extrapolation to the clinical practice.

\section{miRNA AS A THERAPEUTIC TARGET IN THE COLORECTAL CANCER}

One of the major troubles for the CRC treatment is the acquired chemotherapy resistance. As the miRNAs are involved in the cancer progression, they can be considered as prognostic factors or as therapeutic targets (68). For the miRNA inhibition, different tools may be used, such as the miRNA sponges, miRNA masking, antisense oligonucleotides, or molecule inhibitors.

The miRNA sponge is mRNA that has in its sequence multiple tandem binding sites for the targeting of some specific miRNAs. The union between the mRNA and the miRNA produces the selective blockade of a complete family of the associated miRNAs (69). The sponge was tested first in the breast cancer (70), where the mir-21, -155, and -221 were again involved. Shen et al. (71) found the upregulation of the long noncoding RNA cells FBXL19-AS1 in the metastatic CRC. The knockdown of this RNA using the miRNA sponge inhibited the cell migration, proliferation, and invasion in vitro and the tumor growth and metastasis in vivo. The TUSC7, another long noncoding RNA, is a potential tumor suppressor in terms of the CRC. The miR-211 sponge that was observed against the TUSC7 shows a significant downregulation in the CRC tissues compared with the normal tissues, and the survival of the high-expression patients is superior to those with the low expression (72). Other studies have demonstrated the miR-211-3p is an important oncogenic miRNA regarding the CRC (73).

The small molecule inhibitors, such as azobencene, can also be used to modify the miRNA expression (74). Another option is the use of the antisense inhibition of the mature miRNAs (antimiRs) for the blocking of the interaction of the miRNAs with their endogenous mRNA targets. Valeri et al. (75) silenced the miR-135b and the anti-miR-135b by achieving the inhibition of the CRC-cell proliferation. Similar studies show the action by the anti-miRs against the miR-20a (76), miR-21 (77), miR-95 (78), miR-675 (79), and miR-31 (80) in the CRC cell lines. Another strategy involves the stabilization by the synthetic RNA duplexes with chemical modifications (81), as these mimic miRNAs comprise a strand that is identical to that of the miRNA of interest; therefore, their usage in the CRC recovers the expression of the downregulated tumor suppressor, like the miR-26a, $-34 a,-33 a$, or -145 , resulting in a tumor reduction (82). However, the following two drawbacks need to be considered: Some of these miRNAs are very similar to other members of its family, so they can affect the other miRNAs, and the miRNA mimics can produce an immunologic response in the organism (83).

\section{miRNA AS A MARKER OF THE THERAPY RESPONSE}

A low mir-143 expression is a valuable predictive factor for the effectiveness of the capecitabine treatment in the CRC patient (84), and the same applies regarding the miR-31-3p and the miR-31-5p in response to the Cetuximab in the RAS CRC patients (85). A high miR-320e level is associated with the adverse response to the FOLFOX in the stage-III CRC patients (86). Some of the miR-492 polymorphisms in the CRC patients have been associated with a stronger disease progression (87). The miR-129 and the miR-203 are downregulated in the 5-FU resistant cells, and their restitution induces the chemosensitivity (88). Alternatively, high expressions of the miR-192 and the miR-215 have been detected in the 5-FU resistant cells (89), and a high level of the circulating miR-126 is associated with the Bevacizumab-plus XELOX resistance (90). Finally, some miRNAs are also involved in the radiotherapy sensitivity; in particular, the miR-360 has been identified as a 
radiosensitivity regulator. Accordingly, the ionic radiation can decrease the mir-360 expression. When this miRNA is ectopically expressed, it can enhance the ionic-radiationinduced cytotoxicity by the negative regulation of the BCL2L2 and TP53RK expressions (91).

\section{CONCLUSION}

Many miRNAs are deregulated in most of the cancer types including the CRC, driving and modulating their progression. The miRNAs may influence the cancer-stem-cell biology, angiogenesis, epithelial-mesenchymal and mesenchymalepithelial transitions, and/or the drug resistance. Specific patterns of the upregulated and downregulated miRNA have been associated with the CRC diagnosis, prognosis, and therapeutic response. In this review, the miRNA downregulation that occurs in the CRC but not in the normal cells is highlighted, and new strategies of the gene therapy, such as the miRNA sponges, miRNA masking, antisense oligonucleotides, and molecule inhibitors - which are being used to restore their levels, thereby regaining its tumor-suppressor function-are shown. Several miRNAs present the differential expression in the CRC cancer tissues, plasma, or body fluids. These molecules may be used like prognostic and survival biomarkers to activate the tumor-suppression routes or to increase the drug response. In fact, the miRNA panels can be used to quickly identify their circulating concentration, and this has been proposed in relation to the CRC diagnosis and evolution. However, at present the circulating miRNA measurement has rarely been implemented in clinical practice, and the role and function of many miRNAs remain poorly understood. Future research will be necessary to use the miRNAs as a less-invasive technique in the screening for the CRC and to help determine its prognosis.

\section{ACKNOWLEDGEMENTS}

This review was funded by Consejería de Salud de la Junta de Andalucía through the project Pl-0476-2016, Granada University project PP2015-13, and the financial groups 09/112016. We thank Ministerio de Educacion Cultura y Deporte for its research grant (FPU).

\section{CONFLICTS OF INTEREST}

The authors have no conflicting interests.

\section{REFERENCES}

1. Wang F, Fu XD, Zhou $Y$ and Zhang $Y$ (2009) Downregulation of the cyclin E1 oncogene expression by microRNA-16-1 induces cell cycle arrest in human cancer cells. BMB Rep 42, 725-730

2. Hausser J and Zavolan M (2014) Identification and consequences of miRNA-target interactions-beyond repression of gene expression. Nat Rev Genet 15, 599-612

3. Wu BL, Xu LY, Du ZP et al (2011) MiRNA profile in esophageal squamous cell carcinoma: downregulation of miR-143 and miR-145. World J Gastroenterol 17, 79-88

4. Tokarz P and Blasiak J (2012) The role of microRNA in metastatic colorectal cancer and its significance in cancer prognosis and treatment. Acta Biochim Pol 59, 467-474

5. Lee Y, Ahn C, Han J et al (2003) The nuclear RNase III Drosha initiates microRNA processing. Nature 425, 415-419

6. Yi R, Qin Y, Macara IG and Cullen BR (2003) Exportin-5 mediates the nuclear export of pre-microRNAs and short hairpin RNAs. Genes Dev 17, 3011-3016

7. Finnegan EF and Pasquinelli AE (2013) MicroRNA biogenesis: regulating the regulators. Crit Rev Biochem Mol Biol 48, 51-68

8. Hamfjord J, Stangeland AM, Hughes T et al (2012) Differential expression of miRNAs in colorectal cancer: comparison of paired tumor tissue and adjacent normal mucosa using high-throughput sequencing. PLoS One 7, e34150

9. Lu J, Getz G, Miska EA et al (2005) MicroRNA expression profiles classify human cancers. Nature 435, 834-838

10. Weber JA, Baxter DH, Zhang $S$ et al (2010) The microRNA spectrum in 12 body fluids. Clin Chem 56, 1733-1741

11. Chen X, Ba Y, Ma L et al (2008) Characterization of microRNAs in serum: a novel class of biomarkers for diagnosis of cancer and other diseases. Cell Res 18, 997-1006

12. Arends MJ (2013) Pathways of colorectal carcinogenesis. Appl Immunohistochem Mol Morphol 21, 97-102

13. Siegel RL, Miller KD and Jemal A (2016) Cancer statistics, 2016. CA Cancer J Clin 66, 7-30

14. Tarasov VA, Matishov DG, Shin EF et al (2014) [Coordinated aberranit expression of miRNAs in colon cancer]. Genetika 50, 1232-1244

15. Fesler A, Guo S, Liu H, Wu N and Ju J (2017) Overcoming chemoresistance in cancer stem cells with the help of microRNAs in colorectal cancer. Epigenomics 9, 793-796

16. Stiegelbauer V, Perakis S, Deutsch A, Ling H, Gerger A and Pichler M (2014) MicroRNAs as novel predictive biomarkers and therapeutic targets in colorectal cancer. World J Gastroenterol 20, 11727-11735

17. Schee K, Lorenz S, Worren MM et al (2013) Deep sequencing the microRNA transcriptome in colorectal cancer. PLoS One 8, e66165

18. Thiery JP, Acloque $H$, Huang RY and Nieto MA (2009) Epithelial-mesenchymal transitions in development and disease. Cell 139, 871-890

19. Shi $L$, Jackstadt $R$, Siemens $H$, Li $H$, Kirchner $T$ and Hermeking H (2014) p53-induced miR-15a/16-1 and AP4 form a double-negative feedback loop to regulate epithelial-mesenchymal transition and metastasis in colorectal cancer. Cancer Res 74, 532-542

20. Liu R, Lu Z, Gu J et al (2018) MicroRNAs 15A and 16-1 activate signaling pathways that mediate chemotaxis of immune regulatory $\mathrm{B}$ cells to colorectal tumors. Gastroenterology 154, 637-651 e637 
21. Dai L, Wang W, Zhang S et al (2012) Vector-based miR-15a/16-1 plasmid inhibits colon cancer growth in vivo. Cell Biol Int 36, 765-770

22. Cheng HH, Kuo CC, Yan JL et al (2012) Control of cyclooxygenase-2 expression and tumorigenesis by endogenous 5-methoxytryptophan. Proc Natl Acad Sci U S A 109, 13231-13236

23. Young LE, Moore AE, Sokol L, Meisner-Kober $\mathrm{N}$ and Dixon DA (2012) The mRNA stability factor HuR inhibits microRNA-16 targeting of COX-2. Mol Cancer Res 10, $167-180$

24. Chivukula RR, Shi G, Acharya A et al (2014) An essential mesenchymal function for miR-143/145 in intestinal epithelial regeneration. Cell 157, 1104-1116

25. Akao Y, Nakagawa Y and Naoe T (2006) MicroRNAs 143 and 145 are possible common onco-microRNAs in human cancers. Oncol Rep 16, 845-850

26. Takagi $T$, lio A, Nakagawa $Y, N a o e ~ T$, Tanigawa $N$ and Akao $Y$ (2009) Decreased expression of microRNA-143 and -145 in human gastric cancers. Oncology 77, 12-21

27. Szczyrba J, Loprich E, Wach S et al (2010) The microRNA profile of prostate carcinoma obtained by deep sequencing. Mol Cancer Res 8, 529-538

28. Wang W, Ji G, Xiao X et al (2016) Epigenetically regulated miR-145 suppresses colon cancer invasion and metastasis by targeting LASP1. Oncotarget 7, 68674-68687

29. Drebber U, Lay M, Wedemeyer I et al (2011) Altered levels of the onco-microRNA 21 and the tumor-supressor microRNAs 143 and 145 in advanced rectal cancer indicate successful neoadjuvant chemoradiotherapy. Int J Oncol 39, 409-415

30. Li C, Xu N, Li YQ, Wang Y and Zhu ZT (2016) Inhibition of SW620 human colon cancer cells by upregulating miRNA-145. World J Gastroenterol 22, 2771-2778

31. Xu XH, Wu XB, Wu SB, Liu HB, Chen R and Li Y (2014) Identification of miRNAs differentially expressed in clinical stages of human colorectal carcinoma-an investigation in Guangzhou, China. PLoS One 9, e94060

32. Pramanik D, Campbell NR, Karikari C et al (2011) Restitution of tumor suppressor microRNAs using a systemic nanovector inhibits pancreatic cancer growth in mice. Mol Cancer Ther 10, 1470-1480

33. Ibrahim AF, Weirauch U, Thomas M, Grunweller A, Hartmann RK and Aigner A (2011) MicroRNA replacement therapy for miR-145 and miR-33a is efficacious in a model of colon carcinoma. Cancer Res 71, 5214-5224

34. Tanoglu A, Balta AZ, Berber U et al (2015) MicroRNA expression profile in patients with stage II colorectal cancer: A turkish referral center study. Asian Pac J Cancer Prev 16, 1851-1855

35. Roy P, Das S, Auddy RG and Mukherjee A (2014) Engineered andrographolide nanosystems for smart recovery in hepatotoxic conditions. Int J Nanomedicine 9, 4723-4735

36. Kang SM, Lee HJ and Cho JY (2013) MicroRNA-365 regulates NKX2-1, a key mediator of lung cancer. Cancer Lett 335, 487-494

37. Zhang P, Zheng C, Ye H et al (2014) MicroRNA-365 inhibits vascular smooth muscle cell proliferation through targeting cyclin D1. Int J Med Sci 11, 765-770
38. Guo SL, Ye H, Teng $Y$ et al (2013) Akt-p53-miR-365cyclin D1/cdc25A axis contributes to gastric tumorigenesis induced by PTEN deficiency. Nat Commun 4, 2544

39. Lea MA (2010) Recently identified and potential targets for colon cancer treatment. Future Oncol 6, 993-1002

40. Nie J, Liu L, Zheng W et al (2012) microRNA-365, down-regulated in colon cancer, inhibits cell cycle progression and promotes apoptosis of colon cancer cells by probably targeting Cyclin D1 and Bcl-2. Carcinogenesis 33, 220-225

41. Zhou X, Xu X, Wang J, Lin J and Chen W (2015) Identifying miRNA/mRNA negative regulation pairs in colorectal cancer. Sci Rep 5, 12995

42. Papetti M and Augenlicht LH (2011) Mybl2, downregulated during colon epithelial cell maturation, is suppressed by miR-365. Am J Physiol Gastrointest Liver Physiol 301, G508-518

43. Hermeking $H$ (2010) The miR-34 family in cancer and apoptosis. Cell Death Differ 17, 193-199

44. Chang TC, Wentzel EA, Kent OA et al (2007) Transactivation of miR-34a by p53 broadly influences gene expression and promotes apoptosis. Mol Cell 26, 745-752

45. He L, He X, Lim LP et al (2007) A microRNA component of the p53 tumour suppressor network. Nature 447, 1130-1134

46. Jamieson NB, Morran DC, Morton JP et al (2012) MicroRNA molecular profiles associated with diagnosis, clinicopathologic criteria, and overall survival in patients with resectable pancreatic ductal adenocarcinoma. Clin Cancer Res 18, 534-545

47. Gao J, Li N, Dong Y et al (2015) miR-34a-5p suppresses colorectal cancer metastasis and predicts recurrence in patients with stage II/III colorectal cancer. Oncogene 34, 4142-4152

48. Hiyoshi Y, Schetter AJ, Okayama $\mathrm{H}$ et al (2015) Increased microRNA-34b and $-34 \mathrm{c}$ predominantly expressed in stromal tissues is associated with poor prognosis in human colon cancer. PLoS One 10, e0124899

49. Ge P, Yu X, Wang ZC and Lin J (2015) Aberrant methylation of the 1 p36 tumor suppressor gene RIZ1 in renal cell carcinoma. Asian Pac J Cancer Prev 16, 4071-4075

50. Roy S, Levi E, Majumdar AP and Sarkar FH (2012) Expression of miR-34 is lost in colon cancer which can be re-expressed by a novel agent CDF. J Hematol Oncol 5, 58

51. Svoboda M, Izakovicova Holla L, Sefr R et al (2008) Micro-RNAs miR125b and miR137 are frequently upregulated in response to capecitabine chemoradiotherapy of rectal cancer. Int J Oncol 33, 541-547

52. Wang J, Hevi S, Kurash JK et al (2009) The lysine demethylase LSD1 (KDM1) is required for maintenance of global DNA methylation. Nat Genet 41, 125-129

53. Balaguer F, Link A, Lozano JJ et al (2010) Epigenetic silencing of miR-137 is an early event in colorectal carcinogenesis. Cancer Res 70, 6609-6618

54. Chen J, Ding J, Wang Z, Zhu J, Wang X and Du J (2017) Identification of downstream metastasis-associated target 
genes regulated by LSD1 in colon cancer cells. Oncotarget 8, 19609-19630

55. Schaller MD (2001) Paxillin: a focal adhesion-associated adaptor protein. Oncogene 20, 6459-6472

56. Liang L, Li X, Zhang X et al (2013) MicroRNA-137, an HMGA1 target, suppresses colorectal cancer cell invasion and metastasis in mice by directly targeting FMNL2. Gastroenterology 144, 624-635 e624

57. Ng EK, Tsang WP, Ng SS et al (2009) MicroRNA-143 targets DNA methyltransferases $3 \mathrm{~A}$ in colorectal cancer. Br J Cancer 101, 699-706

58. Chen X, Guo X, Zhang H et al (2009) Role of miR-143 targeting KRAS in colorectal tumorigenesis. Oncogene 28, 1385-1392

59. Pichler M, Winter E, Stotz M et al (2012) Down-regulation of KRAS-interacting miRNA-143 predicts poor prognosis but not response to EGFR-targeted agents in colorectal cancer. Br J Cancer 106, 1826-1832

60. Borralho PM, Kren BT, Castro RE, da Silva IB, Steer CJ and Rodrigues CM (2009) MicroRNA-143 reduces viability and increases sensitivity to 5-fluorouracil in HCT116 human colorectal cancer cells. FEBS J 276, 6689-6700

61. Qian X, Yu J, Yin Y et al (2013) MicroRNA-143 inhibits tumor growth and angiogenesis and sensitizes chemosensitivity to oxaliplatin in colorectal cancers. Cell Cycle 12, 1385-1394

62. Liu GH, Zhou ZG, Chen R et al (2013) Serum miR-21 and miR-92a as biomarkers in the diagnosis and prognosis of colorectal cancer. Tumour Biol 34, 2175-2181

63. Kanaan Z, Rai SN, Eichenberger MR et al (2012) Plasma miR-21: a potential diagnostic marker of colorectal cancer. Ann Surg 256, 544-551

64. Aherne ST, Madden SF, Hughes DJ et al (2015) Circulating miRNAs miR-34a and miR-150 associated with colorectal cancer progression. BMC Cancer 15, 329

65. Imaoka H, Toiyama Y, Fujikawa $\mathrm{H}$ et al (2016) Circulating microRNA-1290 as a novel diagnostic and prognostic biomarker in human colorectal cancer. Ann Oncol 27, 1879-1886

66. Kanaan Z, Roberts H, Eichenberger MR et al (2013) A plasma microRNA panel for detection of colorectal adenomas: a step toward more precise screening for colorectal cancer. Ann Surg 258, 400-408

67. Ahmed FE (2014) miRNA as markers for the diagnostic screening of colon cancer. Expert Rev Anticancer Ther 14, 463-485

68. Yi R, Li Y, Wang FL, Miao G, Qi RM and Zhao YY (2016) MicroRNAs as diagnostic and prognostic biomarkers in colorectal cancer. World J Gastrointest Oncol 8, 330-340

69. Ebert MS and Sharp PA (2010) MicroRNA sponges: progress and possibilities. RNA 16, 2043-2050

70. Jung J, Yeom C, Choi YS et al (2015) Simultaneous inhibition of multiple oncogenic miRNAs by a multi-potent microRNA sponge. Oncotarget 6, 20370-20387

71. Shen B, Yuan Y, Zhang Y et al (2017) Long non-coding RNA FBXL19-AS1 plays oncogenic role in colorectal cancer by sponging miR-203. Biochem Biophys Res Commun 488, 67-73

72. Xu J, Zhang R and Zhao J (2017) The Novel Long Noncoding RNA TUSC7 Inhibits Proliferation by
Sponging MiR-211 in Colorectal Cancer. Cell Physiol Biochem 41, 635-644

73. Wu X, He X, Li S, Xu X, Chen X and Zhu H (2016) Long non-coding RNA ucoo2kmd.1 regulates CD44-dependent cell growth by competing for miR-211-3p in colorectal cancer. PLoS One 11, e0151287

74. Gumireddy K, Young DD, Xiong X, Hogenesch JB, Huang $Q$ and Deiters A (2008) Small-molecule inhibitors of microrna miR-21 function. Angew Chem Int Ed Engl 47, 7482-7484

75. Valeri N, Braconi C, Gasparini P et al (2014) MicroRNA-135b promotes cancer progression by acting as a downstream effector of oncogenic pathways in colon cancer. Cancer Cell 25, 469-483

76. Chai H, Liu M, Tian R, Li X and Tang H (2011) miR-20a targets BNIP2 and contributes chemotherapeutic resistance in colorectal adenocarcinoma SW480 and SW620 cell lines. Acta Biochim Biophys Sin (Shanghai) $43,217-225$

77. Valeri N, Gasparini P, Braconi C et al (2010) MicroRNA-21 induces resistance to 5 -fluorouracil by down-regulating human DNA MutS homolog 2 (hMSH2). Proc Natl Acad Sci U S A 107, 21098-21103

78. Huang Z, Huang S, Wang Q et al (2011) MicroRNA-95 promotes cell proliferation and targets sorting Nexin 1 in human colorectal carcinoma. Cancer Res 71, 2582-2589

79. Tsang WP, Ng EK, Ng SS et al (2010) Oncofetal $\mathrm{H} 19$-derived miR-675 regulates tumor suppressor $\mathrm{RB}$ in human colorectal cancer. Carcinogenesis 31, 350-358

80. Wang CJ, Stratmann J, Zhou ZG and Sun XF (2010) Suppression of microRNA-31 increases sensitivity to 5-FU at an early stage, and affects cell migration and invasion in HCT-116 colon cancer cells. BMC Cancer 10, 616

81. van Rooij E and Kauppinen S (2014) Development of microRNA therapeutics is coming of age. EMBO Mol Med 6, 851-864

82. Christopher AF, Kaur RP, Kaur G, Kaur A, Gupta V and Bansal P (2016) MicroRNA therapeutics: Discovering novel targets and developing specific therapy. Perspect Clin Res 7, 68-74

83. Kawai T and Akira S (2010) The role of patternrecognition receptors in innate immunity: update on Toll-like receptors. Nat Immunol 11, 373-384

84. Simmer F, Venderbosch S, Dijkstra JR et al (2015) MicroRNA-143 is a putative predictive factor for the response to fluoropyrimidine-based chemotherapy in patients with metastatic colorectal cancer. Oncotarget 6 , 22996-23007

85. Mlcochova J, Faltejskova-Vychytilova P, Ferracin $M$ et al (2015) MicroRNA expression profiling identifies miR-31-5p/3p as associated with time to progression in wild-type RAS metastatic colorectal cancer treated with cetuximab. Oncotarget 6, 38695-38704

86. Perez-Carbonell L, Sinicrope FA, Alberts SR et al (2015) $\mathrm{MiR}-320 \mathrm{e}$ is a novel prognostic biomarker in colorectal cancer. Br J Cancer 113, 83-90

87. Lee HC, Kim JG, Chae YS et al (2010) Prognostic impact of microRNA-related gene polymorphisms on survival of patients with colorectal cancer. J Cancer Res Clin Oncol $136,1073-1078$ 
88. Li T, Gao F and Zhang XP (2015) miR-203 enhances chemosensitivity to 5 -fluorouracil by targeting thymidylate synthase in colorectal cancer. Oncol Rep 33, 607-614

89. Boni V, Bitarte N, Cristobal I et al (2010) miR-192/miR-215 influence 5-fluorouracil resistance through cell cyclemediated mechanisms complementary to its posttranscriptional thymidilate synthase regulation. Mol Cancer Ther 9, 2265-2275

90. Hansen TF, Carlsen AL, Heegaard NH, Sorensen FB and Jakobsen A (2015) Changes in circulating microRNA-126 during treatment with chemotherapy and bevacizumab predicts treatment response in patients with metastatic colorectal cancer. Br J Cancer 112, 624-629

91. Zhang $\mathrm{Y}, \mathrm{Yu}$ J, Liu $\mathrm{H}$ et al (2015) Novel epigenetic CREB-miR-630 signaling axis regulates radiosensitivity in colorectal cancer. PLoS One 10, e0133870

92. Diaz R, Silva J, Garcia JM et al (2008) Deregulated expression of miR-106a predicts survival in human colon cancer patients. Genes Chromosomes Cancer 47, 794-802

93. Sakaguchi M, Hisamori S, Oshima N, Sato F, Shimono Y and Sakai Y (2016) miR-137 Regulates the tumorigenicity of colon cancer stem cells through the inhibition of
DCLK1. Mol Cancer Res 14, 354-362

94. Wang CJ, Zhou ZG, Wang L et al (2009) Clinicopathological significance of microRNA-31, -143 and -145 expression in colorectal cancer. Dis Markers 26, 27-34

95. Wang B, Li W, Liu H et al (2014) miR-29b suppresses tumor growth and metastasis in colorectal cancer via downregulating Tiam1 expression and inhibiting epithelialmesenchymal transition. Cell Death Dis 5, e1335

96. Li J, Mao X, Wang X and Miao G (2017) miR-433 reduces cell viability and promotes cell apoptosis by regulating MACC1 in colorectal cancer. Oncol Lett 13, 81-88

97. Guo ST, Jiang CC, Wang GP et al (2013) MicroRNA-497 targets insulin-like growth factor 1 receptor and has a tumour suppressive role in human colorectal cancer. Oncogene 32, 1910-1920

98. He D, Wang J, Zhang C et al (2015) Down-regulation of miR-675-5p contributes to tumor progression and development by targeting pro-tumorigenic GPR55 in non-small cell lung cancer. Mol Cancer 14, 73

99. Wang Q, Huang Z, Ni S et al (2012) Plasma miR-601 and miR-760 are novel biomarkers for the early detection of colorectal cancer. PLoS One 7, e44398 